\title{
Potential Applications of PET/MR Imaging in Cardiology
}

\author{
Osman Ratib ${ }^{1}$ and René Nkoulou ${ }^{1,2}$ \\ ${ }^{1}$ Division of Nuclear Medicine and Molecular Imaging, University Hospital of Geneva, Geneva, Switzerland; and ${ }^{2}$ Division of \\ Cardiology, University Hospital of Geneva, Geneva, Switzerland
}

Recent advances in hybrid PET/MR imaging have opened new perspectives for cardiovascular applications. Although cardiac MR imaging has gained wider adoption for routine clinical applications, PET images remain the reference in many applications for which objective analysis of metabolic and physiologic parameters is needed. In particular, in cardiovascular diseases-more specifically, coronary artery disease - the use of quantitative and measurable parameters in a reproducible way is essential for the management of therapeutic decisions and patient follow-up. Functional MR images and dynamic assessment of myocardial perfusion from transit of intravascular contrast medium can provide useful criteria for identifying areas of decreased myocardial perfusion or for assessing tissue viability from late contrast enhancement of scar tissue. PET images, however, will provide more quantitative data on true tissue perfusion and metabolism. Quantitative myocardial flow can also lead to accurate assessment of coronary flow reserve. The combination of both modalities will therefore provide complementary data that can be expected to improve the accuracy and reproducibility of diagnostic procedures. But the true potential of hybrid PET/MR imaging may reside in applications beyond the domain of coronary artery disease. The combination of both modalities in assessment of other cardiac diseases such as inflammation and of other systemic diseases can also be envisioned. It is also predicted that the 2 modalities combined could help characterize atherosclerotic plaques and differentiate plaques with a high risk of rupture from stable plaques. In the future, the development of new tracers will also open new perspectives in evaluating myocardial remodeling and in assessing the kinetics of stem cell therapy in myocardial infarction. New tracers will also provide new means for evaluating alterations in cardiac innervation, angiogenesis, and even the assessment of reporter gene technologies. The fusion of 2 potentially competing modalities can certainly offer the best of each modality in a single procedure. The impact of such advanced technology in routine clinical practice will still need to be demonstrated. Beyond the expected improvement in patient management and the potential impact on patient outcome, PET/MR imaging will also need to establish its medicoeconomic justification in an era of health-care economic restrictions.

Key Words: hybrid PET/MRI; nuclear cardiology, cardiac PET

J Nucl Med 2014; 55:40S-46S

DOI: 10.2967/jnumed.113.129262

\footnotetext{
Received Nov. 5, 2013; revision accepted Mar. 11, 2014.

For correspondence or reprints contact: Osman Ratib, Division of Nuclear Medicine and Molecular Imaging, Department of Medical Imaging and Information Sciences, University Hospital of Geneva, 24 rue Micheli-du-Crest, 1205 Geneva, Switzerland.

E-mail: osman.ratib@hcuge.ch

Published online Apr. 24, 2014.

COPYRIGHT (c) 2014 by the Society of Nuclear Medicine and Molecular Imaging, Inc.
}

$\mathbf{T}$ he emergence of hybrid PET/CT more than a decade ago has changed the perspective on the applicability of PET in clinical routines. The added value of anatomic localization and morphologic characterization provided by CT images allowed a better confidence in PET image interpretation and diagnostic accuracy, yielding a wider adoption of PET investigation in clinical practice. Although mostly driven by oncologic applications, the wider availability of PET/CT scanners has led to its broader access for other applications such as cardiology and neurology. Despite limitations in reimbursements, the progressive increase in PET/CT applications for the assessment of cardiovascular diseases has made its way among the wide range of diagnostic imaging modalities in cardiology. The rapid evolution of hybrid PET/CT with multidetector CT scanners that allow high-definition dynamic imaging of the heart and coronary vessels has opened a new perspective on cardiac imaging by providing combined anatomic and functional evaluation of coronary disease and alterations in cardiac function (1). At the same time, MR imaging technology has evolved, with new advanced imaging methods that are applicable to diagnostic and functional analysis of the heart and great vessels. Although high-definition cine sequences provided high-definition dynamic visualization of the heart and valvular structures, first-pass imaging of contrast medium transit through the myocardium was shown to depict stress-induced alteration in myocardial blood flow, differentiating between normal and hypoperfused myocardium (2). In some ways, MR imaging provides additional and valuable information that $\mathrm{CT}$ cannot provide, but it lacks the temporal and spatial resolution of CT for the accurate visualization of the coronary arteries. The unmatched accuracy of PET for the measurement of myocardial perfusion and the assessment of myocardial viability has contributed to the competition between PET/CT and MR imaging for investigating coronary artery disease (CAD) and other cardiovascular afflictions. The higher radiation exposure and limited reimbursement policies of PET/CT have given MR imaging a significant advantage, leading to a broader adoption in clinical practice. It may therefore be foreseeable that hybrid PET/MR imaging could benefit from the best of the 2 modalities and lead to a better synergy between the metabolic capabilities of PET tracers and the functional and anatomic performance of MR imaging.

The aim of this article is to review the early observations and clinical applications of hybrid PET/MR imaging in the evaluation of cardiovascular disease and CAD in particular. This article also highlights other potential applications of hybrid PET/MR imaging beyond the scope of CAD that may foresee the application of this technique in other cardiovascular uses such as the evaluation of vascular atherosclerotic plaques and, through the development of new radiotracers, the assessment of alterations in neurotransmitters, the evaluation of mechanisms of angiogenesis, and even the follow-up of stem cell therapy. 


\section{GENERAL PERSPECTIVES ON PET/MR IMAGING APPLICATIONS}

The first difficulty in assessing the impact and potential perspective of hybrid PET/MR imaging is the diversity and technical differences of the first devices that appeared on the market. Although the first generation included separate or coplanar PET and MR imaging scanners, they were based on state-of-the-art, photomultiplier-based PET scanners that offered the highest definition and best technical performance, allowing in particular time-of-flight image acquisition. The limitation of these early hybrid devices is that they did not allow simultaneous acquisition of images from both modalities and they required sequential acquisitions. In the next generation of integrated PET/MR imaging devices, avalanche photodiode PET detector modules are incorporated inside the bore of the MR scanner. Despite the more limited temporal performance and spatial resolution of the first generation of avalanche photodiodes compared with standard photomultiplier detectors, the integration inside the MR scanner allowed simultaneous acquisition of PET and MR images of a given segment of the body (3). A second generation of integrated devices using higherperformance solid-state photomultipliers that provide higher sensitivity and temporal resolution and allow time-of-flight imaging are arriving on the market. The rapid change in technology and the variety of devices installed restrict the ability to gather sufficient clinical experience to properly evaluate the added value of hybrid acquisition over traditional combinations of PET and MR imaging data that are acquired on separate devices at different points in time.

However, in evaluating the potential impact of these new devices, one should emphasize the strong contribution of the ability of PET to provide objective and quantitative measurements that MR imaging lacks. It is therefore essential for cardiovascular application to benefit from the best possible performance of the PET component (4). Also, given the wealth of diagnostic and functional information that MR images can provide, comparison with conventional PET/CT scanners is relatively difficult.

Apart from the technical aspects of the different solutions for integrating PET and MR modalities while maintaining the best performance of each modality without interference between them, the main challenges in applying PET/MR imaging for cardiovascular applications are the development of reliable attenuation correction techniques, the design and implementation of optimized protocols, and efficient patient workflow. An example of cardiac viability assessment using a coplanar PET/MR imaging system is displayed in Figure 1. Another challenge is the need to bring together a multidisciplinary team that can provide the necessary sets of expertise and skills for designing acquisition protocols and performing complex analysis and image interpretation in this uncharted environment, as discussed in recent articles by Rischpler et al. (5) and Ratib et al. (6).

The general limitations of patients who cannot be imaged with a PET/MR imaging device are the same as those with known contraindications to MR imaging, including patients who have claustrophobia; patients with a pacemaker, metallic clips, or mechanical valves; and large patients because of the small scanner apertureonly about $60 \mathrm{~cm}$ in diameter for the current generation of PET/MR imaging scanners.

Lack of reimbursement of cardiac MR imaging and PET procedures can lead to the same restrictions as were known for PET/CT studies that were not endorsed by insurance companies and third-party payers.

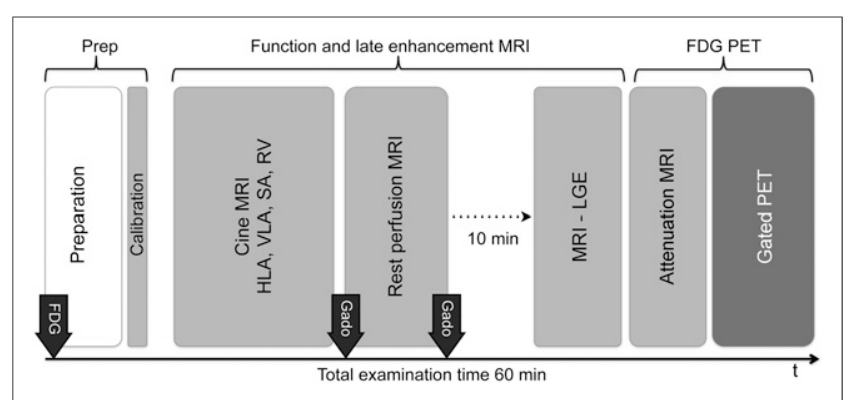

FIGURE 1. Protocol for hybrid PET/MR imaging evaluation of myocardial viability. Protocol includes sequences of cine MR imaging for evaluation of alteration in regional wall motion, rest MR perfusion, and late enhancement scans, as well as gated ${ }^{18} \mathrm{~F}-\mathrm{FDG}$ PET sequences. HLA = horizontal long axis; VLA = vertical long axis; $S A=$ short axis; $R V=$ right ventricle.

\section{WHAT MR IMAGING CAN BRING TO CARDIAC PET IMAGING}

$\mathrm{CAD}$ is among the major public health issues of our Western societies and is still largely perceived as a consequence of the incremental occlusion of coronary arteries through progressive vascular stenosis that can be diagnosed by coronary angiography and treated by percutaneous or surgical coronary revascularization. Today, an unreasonably large number of symptomatic and asymptomatic at-risk patients with suspected CAD will undergo coronary angiography with normal findings (7). Also, recent large randomized trials have emphasized the limitations of coronary arteriography studies that lead to therapeutic interventions based only on the morphologic severity of epicardial coronary stenosis; these trials advocate for additional proof of hemodynamically significant stress-induced reduction in coronary flow before any therapeutic interventions are undertaken $(8-10)$.

The myocardial perfusion imaging technique using contrastenhanced cardiac MR imaging (CMR) of the myocardium is an established modality with numerous well-documented studies describing diagnostic accuracy comparable to that of SPECT, which is more susceptible to soft-tissue attenuation and carries the disadvantage of additional radiation exposure to the patient $(11,12)$. On the basis of these observations, CMR is generally regarded as a safer procedure than traditional nuclear medicine imaging techniques, despite some preliminary studies that show associated DNA damages after CMR (13) in similar proportions to those already known with $x$-ray and $\gamma$-ray exposure and that document infrequent, gadolinium-related nephrogenic systemic fibrosis. The major advantages of CMR remain its excellent soft-tissue contrast, higher spatial resolution, and the possibility of complementing the myocardial perfusion examination with additional diagnostic criteria regarding cardiac volume and ventricular function, soft-tissue characterization, and valvular morphology, in addition to its ability to delineate scar tissue and differentiate viable from nonviable myocardium after myocardial infarction. Most CMR examinations are performed and validated on systems with 1.5-T magnetic field strength. Current MR scanners used in hybrid PET/MR imaging are based on a 3.0-T field strength, enabling a better signal-to-noise ratio at the expense of more frequent susceptibility effects and radiofrequency inhomogeneities that require finely tuned acquisition parameters and higher expertise in analyzing the results and avoiding false interpretations (14). The prerequisites of fine tuning and higher expertise might partly explain the relative lower performance of CMR as compared with SPECT in assessing myocardial perfusion in controlled multicentric studies (15). The general limitations of perfusion 
CMR are limited spatial coverage and low first-pass extraction of contrast medium, which lead to the underestimation of flow differences that have so far prevented clinically meaningful evaluation of absolute myocardial blood flow (16). To date, direct comparison studies between CMR and PET perfusion are relatively limited.

Although different imaging techniques are available for the evaluation of myocardial perfusion, PET remains the reference technique that has been extensively validated for the accurate detection of hemodynamically significant coronary stenosis as compared with invasive coronary angiography; PET also provides accurate and reproducible evaluation of absolute myocardial blood flow at rest and under stress conditions, as well as an accurate estimate of coronary flow reserve (17). The different PET perfusion tracers are either freely diffusible $\left({ }^{15} \mathrm{O}\right.$-water $)$ or metabolized $\left({ }^{13} \mathrm{~N}\right.$-ammonia and $\left.{ }^{82} \mathrm{Rb}\right)$ with important first-pass activity and retention; they also provide predictable kinetic models that can be applied using an arterial input function gathered through dynamic acquisition of the whole heart. Absolute measures of myocardial blood flow and coronary flow reserve, defined as the ratio of stress to rest myocardial blood flow, have been shown to assist in the diagnosis of CAD, complementing the relative comparison of regional blood flow in patients who have a normal relative flow pattern, as might occur during balanced reduction of regional perfusion in the context of diffuse coronary disease (18). Myocardial activity and coronary flow reserve also detect the preclinical alteration of coronary vasomotion that might be properly addressed by correcting coronary risk factors (19).

Some limitations of the assessment of myocardial perfusion with PET are related to the volatility of perfusion PET tracers, the relatively moderate spatial resolution, and the associated albeit reduced radiation to the patient as compared with myocardial perfusion obtained by SPECT. The very short physical half-life of some PET perfusion tracers requires on-site cyclotron or rubidium generators that represent a high initial cost compared with SPECT perfusion tracers. This factor, along with limited reimbursement by insurance companies outside the United States, has limited the wider use of PET perfusion studies despite evidence of a better diagnostic accuracy and cost-effectiveness as compared with other modalities. However, new promise for future PET perfusion studies is offered by new ${ }^{18} \mathrm{~F}$-labeled perfusion tracers arriving soon on the market that have a 110-min half-life, high first-pass extraction, and nearly linear flow-related uptake (20). The quantitative assessment of the transmural extension of perfusion defects with PET perfusion tracers has been well demonstrated (21). However, the detection of subtle subendocardial ischemia may suffer from the limited resolution of PET. The new generation of PET scanners with improved spatial resolution and new ${ }^{18} \mathrm{~F}$ labeled flow tracers is expected to further improve the performance of PET for the detection and quantification of regional myocardial ischemia. These benefits can probably be demonstrated only in prospective studies using hybrid systems that can perform coregistration of the 2 modalities with a high degree of accuracy and can benefit from the high resolution and morphologic localization of regional alteration that MR imaging can provide. Furthermore, PET perfusion studies that may possibly detect preclinical vasomotor coronary dysfunction might also add a new perspective for screening in symptomatic patients, provided that global radiation exposure is significantly reduced when using MR imaging instead of CT for attenuation correction (22).

Another domain of interest is patients with ischemia-related left ventricular dysfunction in whom improvement in myocardial contractility can be expected after revascularization procedures, provided that sufficient residual myocardial viability has been demonstrated (23). A review of the diagnostic performance of different imaging modalities in unveiling myocardial viability is beyond the scope of the present review (24), but viability is becoming a highly relevant condition for defining the appropriateness of applying interventional techniques for the revascularization of chronic totally occluded coronary arteries, particularly in the scope of the increased prevalence of chronic ischemic cardiomyopathy in the aging population. Preliminary results of recent controlled trials investigating the role of different clinical pathways that integrate a viability imaging procedure before revascularization outline the difficulty in recruitment and implementation of complex therapeutic procedures in such inhomogeneous groups of patients $(25,26)$. Recent recommendations still support the need for an objective, selective therapeutic approach in patients who have proven myocardial viability and can benefit from such treatments. However, the current limitations of PET metabolic imaging using ${ }^{18}$ F-FDG (sensitivity, 92\%; specificity, 63\%) or CMR morphologic and functional imaging based on late gadolinium enhancement (LGE), combined with dobutamine-induced dysfunction in left ventricular contractility (sensitivity, $84 \%$; specificity, 63\%) must be considered (27). The combination of both modalities can yield more accurate and objective assessment of viable and functionally recoverable myocardial tissue. In this group of patients, the limited risk of radiation exposure from PET is extremely low compared with the relevance of the impact of effective therapeutic intervention and the long-term risks of chronic and progressive cardiac dysfunction. The limitations of PET in this regard are due to the natural inhomogeneity in myocardial substrate use that requires special and careful patient preparation using glucose infusion, insulin, or acipimox before the study, which is often quite challenging in diabetic patients (28). The moderate spatial resolution of conventional PET systems is also a technical limitation for discriminating subendocardial scarring from viable myocardium with a mildly altered metabolic uptake. Keep in mind that ${ }^{18} \mathrm{~F}-\mathrm{FDG}$ is a nonspecific metabolic tracer, the uptake of which can be increased in areas of inflammations (as in recent infarction and cardiac inflammatory diseases) as well as in chronically ischemic regions that will shift to high ${ }^{18}$ F-FDG metabolic activity compared with adjacent normal or reduced uptake in nonischemic myocardium (Fig. 2). The accurate interpretation of findings of cardiac PET examinations requires careful analysis of a variety of conditions that can interfere with the physiologic relationship between metabolic abnormalities and underlying coronary disease, including the knowledge of coronary occlusion, coronary distribution, and left ventricular functional parameters, all of which can be extracted from CMR studies. This need was recently highlighted by a study by Abraham et al. (29) describing an improved patient outcome in centers that have highly specialized cardiac expertise compared with standard-care facilities. Using CMR data systematically to complement viability PET data is undoubtedly opening new perspectives for better management of CAD patients, but it can also raise some legitimate questions in the added complexity of the uncertainty of results with combined modalities. The complementary findings gathered by each modality can certainly increase the physician's confidence in identifying scar tissue in regions with a matched reduced perfusion-metabolic pattern and extensive LGE $(>75 \%)$. Similarly, those areas with mismatched metabolic patterns and an absence of significant LGE $(<25 \%)$ can identify segments with viable myocardium and help predict the recoverable contractility after optimal treatment (Figs. 3 and 4). 

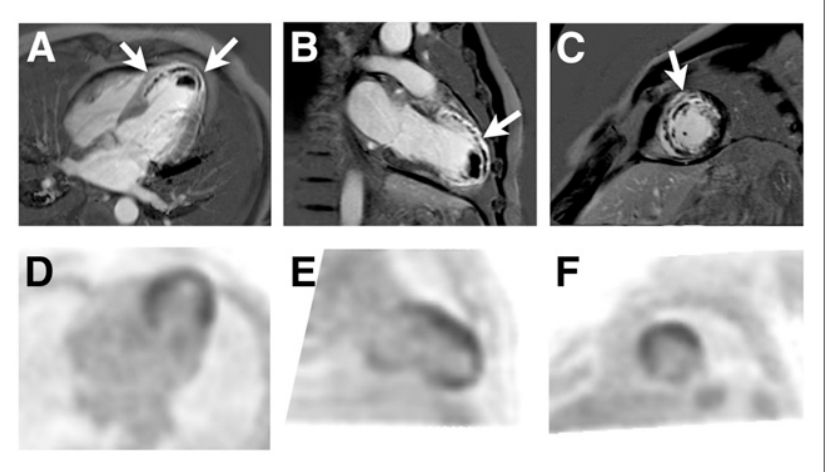

FIGURE 2. Patient with recent ST-segment myocardial infarction who underwent acute percutaneous coronary intervention of proximal left anterior descending coronary artery and showed angiographic finding of chronic total occlusion of the right coronary artery. Viability PET/MR imaging performed $3 \mathrm{~d}$ after acute event shows extensive transmural late enhancement of anteroapical wall on MR imaging (arrows on A, horizontal long-axis view; B, vertical long-axis view; and C, short-axis view) and unexpectedly high ${ }^{18} \mathrm{~F}-\mathrm{FDG}$ uptake in same territory $(\mathrm{D}, \mathrm{E}$, and $F$ respectively). Although this uptake could suggest viable myocardium, its high intensity and mismatch with myocardial scarring shown on MR imaging suggest possible inflammatory tissue activity in infarcted segment in this PET scan performed only $3 \mathrm{~d}$ after acute event.

However, discrepant findings between the 2 modalities with a mismatch between molecular (perfusion-metabolism) and functional (LGE, low dobutamine-enhanced contractility) evaluations is relatively frequent, occurring in $20 \%-36 \%$ of cases, and it remains unclear how these findings should be considered for the clinical management of these patients (30). More longitudinal controlled studies are needed to better understand the impact of these unmatched regional alterations on patient outcome and whether patients may require revascularization interventions. Another aspect of MR imaging that still remains to be investigated is the value of a rest perfusion study that can be acquired before LGE and its value in place of an additional PET or SPECT perfusion study for the evaluation of myocardial perfusion patterns at rest.

However, one should still consider that for both early CAD detection and characterization of advanced CAD, CT will probably

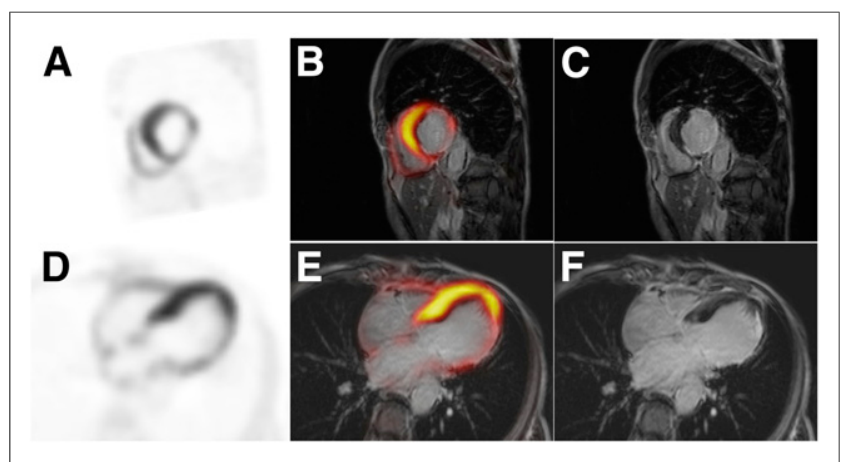

FIGURE 3. Patient admitted for recent-onset dyspnea on exertion whose cardiac ultrasound showed severely decreased left ventricular ejection fraction (30\%) with inferior-to-posterolateral akinesia. Perfusion SPECT showed fixed defect in same territory. Viability PET/MR imaging study showed significantly decreased metabolic activity of posterolateral wall on short-axis (A) and 4-chamber (D) views. These areas of decreased tracer uptake match perfectly segments showing late gadolinium enhancement on MR images ( $C$ and $F$, respectively), as shown on fused images ( $B$ and $E$ ).

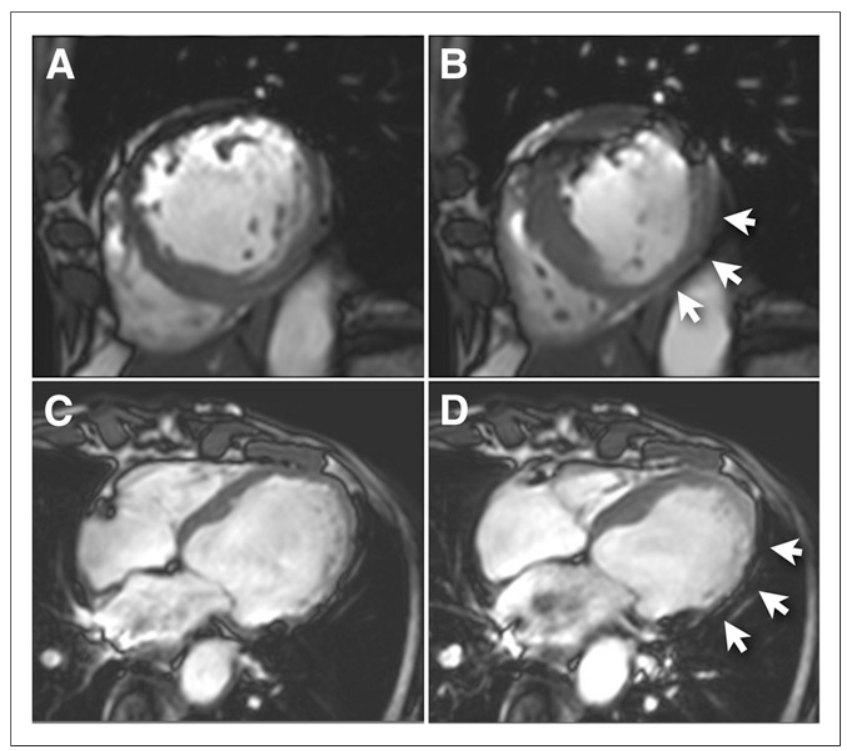

FIGURE 4. Cine MR images of same patient as in Figure 3 obtained in midventricular short-axis plane during diastole (A) and systole (B) show severe hypokinesis of lateral wall (arrows, B). Images obtained in 4-chamber view in diastole (C) and systole (D) show normal septal wall thickening and extended akinesis of lateral wall (arrows, D).

still play an important role when associated with PET and its ability to quantify coronary calcification as a prognostic marker as well as its ability to visualize coronary anatomy that is currently not feasible with MR imaging (31). With recent improvements in CT technology and processing that allow very low-dose coronary CT angiography and functional assessment of ventricular function, along with the potential capabilities of future systems for evaluating myocardial perfusion and delayed enhancement with exquisite nonisotropic resolution, PET/CT will continue to be a competing hybrid modality for the assessment of CAD (32).

\section{WHAT PET CAN BRING TO OTHER CMR APPLICATIONS}

\section{Inflammatory Cardiomyopathies}

The high spatial resolution and soft-tissue characterization of cardiac MR imaging has shown a great potential in the identification and evaluation of myocardial alteration related to inflammatory cardiomyopathies such as sarcoidosis. Sarcoidosis is a granulomatous disease of unknown etiology that most commonly affects the lungs and mediastinal lymph nodes, with cardiac involvement responsible for $30 \%-85 \%$ of deaths from an atrioventricular conduction block or ventricular arrhythmia (33) related to granulomatous infiltration or subsequent fibrotic scars. The diagnosis of cardiac sarcoidosis is often confirmed invasively by endomyocardial biopsy performed through right heart catheterization. Since cardiac involvement is inhomogeneous, the histologic confirmatory diagnostic and procedural risks are related to the number and location of samples, and there is a high prevalence of negative findings. An accurate noninvasive imaging technique is therefore necessary to exclude cardiac involvement or to help guide biopsy in segments with suspected infiltration. Cardiac MR imaging has emerged as the current modality of choice for the diagnosis of cardiac involvement, with regional functional assessment and T2-weighted sequences for the detection of edema and inflammation complementing the LGE sequences for the identification of fibrotic tissue (34). Some initial case reports demonstrat- 


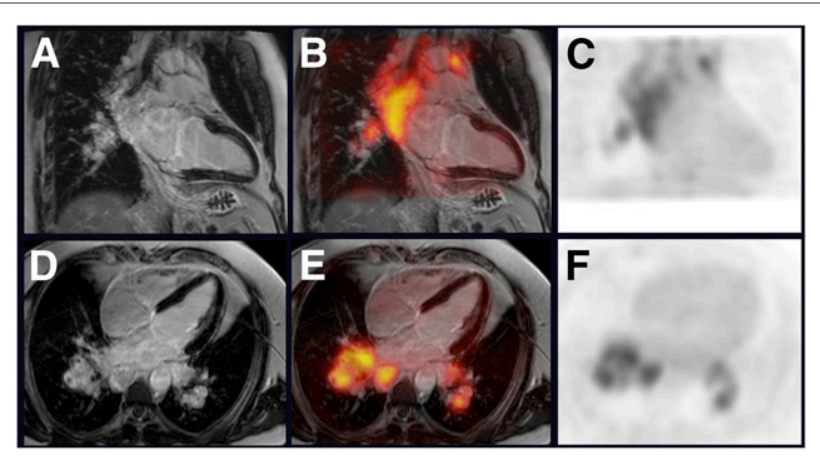

FIGURE 5. Patient with pulmonary sarcoidosis who was investigated for possible cardiac extension. Late-enhancement MR images (A, C, and D) obtained in vertical long-axis (A) and 4-chamber (D) views show no evidence of myocardial gadolinium enhancement. ${ }^{18} \mathrm{~F}-\mathrm{FDG}$ PET images obtained in same planes ( $\mathrm{C}$ and $\mathrm{F}$ ) show no myocardial uptake. Fused images ( $B$ and $E$ ) show no evidence of myocardial lesions and show high ${ }^{18} \mathrm{~F}-\mathrm{FDG}$ uptake only in massively enlarged mediastinal lymph nodes.

ing the value of ${ }^{18}$ F-FDG PET studies for identifying cardiac sarcoidosis (35) have been followed by more systematic studies of PET perfusion and metabolic patterns of cardiac involvement (36). Several prospective studies documented the diagnostic and prognostic value of ${ }^{18}$ F-FDG PET in cardiac sarcoidosis $(37,38)$. Correlative studies of CMR and PET showed comparable accuracy of both modalities in detecting myocardial lesions, with better correlation of abnormal PET findings to elevated angiotensin-conversing enzyme levels but relatively poor specificity (only 38.5\%) (39). These results may also be related to suboptimal patient preparation and insufficient cardiac metabolic suppression protocols, including extended fasting; a low-protein, high-fat diet; or heparin infusion. Also, different distributions of abnormal findings using CMR and PET were observed due to concomitant coronary artery disease and other myocardial inflammatory processes. However, simultaneous PET/MR imaging in patients with suspected cardiac sarcoidosis benefits from the improved spatial resolution of LGE sequences to describe areas of myocardial infiltration that can be further characterized by their metabolic activity on PET images as inflammatory or scar-related, complementing the less specific MR sequences. PET also provides a more comprehensive whole-body assessment of extracardiac involvement, including pulmonary or neurologic extensions of the disease that are less accessible by MR imaging alone. Some examples of patients with suspected cardiac sarcoidosis from our institution are presented in Figures 5 and 6.

\section{Other Infiltrative Cardiomyopathies}

The recent development of PET tracers for amyloid plaque identification in Alzheimer disease has generated potential interest in the evaluation of cardiac amyloidosis, a condition that is traditionally diagnosed using MR imaging (40). MR images usually show unspecific focal LGE abnormalities related to minor fibrosis in the myocardium that are only a small expression of the disease in comparison with the massive amyloid infiltration most commonly affecting the subendocardial wall. Whereas the relatively modest scarring involvement in relation to amyloid infiltration is a limitation to diagnostic sensitivity, the subendocardial localization of such findings can also be potentially mistaken for existing myocardial infarction islands in CAD, thereby reducing the specificity of such findings for the confirmation of amyloidosis. The heart normally exhibits a low concentration of amyloid, and preliminary studies using amyloid PET tracers have shown promising results in the diagnosis of cardiac infiltration $(41-43)$.

\section{OTHER POTENTIAL APPLICATIONS OF HYBRID CARDIAC PET/ MR IMAGING}

The ability to acquire high-definition MR images with tissue characterization and functional capability, combined with metabolic information provided by current and future PET tracers, opens new perspectives in the clinical application of hybrid PET/ MR imaging beyond the assessment of coronary artery disease and myocardial viability.

One of the most promising applications is the evaluation and characterization of atherosclerotic plaques in peripheral vessels and potentially also in coronary vessels. The ability to detect highrisk vascular plaques even in the absence of significant luminal obstruction could help identify those plaques that could potentially rupture and cause future vascular events $(44,45)$. The causes of plaque instability and high rupture risk are believed to be related to inflammatory processes and the accumulation of macrophages in the lipid core of vessel plaques (46). Although inflammation can be detected with ${ }^{18} \mathrm{~F}$-FDG PET, more specific tracers are also being explored, such as tracers of intraplaque angiogenesis that can identify the proliferation of medial vasa vasorum, which has also been shown to occur in rapid plaque growth and plaque rupture. Therefore, PET imaging of inflammation and angiogenesis within atherosclerotic plaques may be useful to identify high-risk plaques and could also allow the monitoring of antiatherosclerotic therapies (47). Among the new tracers being explored for the detection of plaque inflammation and angiogenesis is ${ }^{18} \mathrm{~F}$-galactoRGD, which binds with high specificity to $\alpha_{v} \beta_{3}$ integrin, a cell surface glycoprotein receptor that is highly expressed during

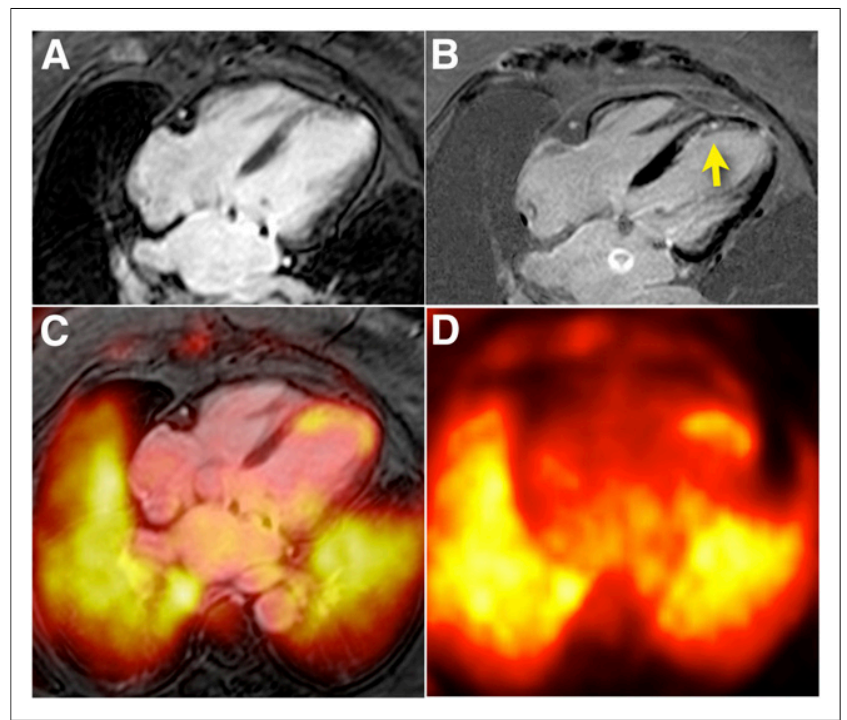

FIGURE 6. Patient with history of coronary artery disease who was investigated for possible myocardial extension of pulmonary sarcoidosis. Gated cine (A) and contrast-enhanced MR (B) images show patchy subendocardial late-enhancement pattern of septa-apical wall (arrow, B). ${ }^{18} \mathrm{~F}-\mathrm{FDG}$ PET image (D) shows increased uptake in same territory compared with surrounding depressed myocardial uptake $(C=$ fusion of $A$ and D). Although this finding suggests subendocardial myocardial scarring with residual viable but hibernating myocardium and anaerobic metabolism, differential diagnosis remains possible focal sarcoidosis. High ${ }^{18} \mathrm{~F}-\mathrm{FDG}$ uptake is seen in lung, consistent with active sarcoidosis. 
angiogenesis (48). Although these early studies are promising, further preclinical and clinical studies are needed to characterize its uptake in plaques with high degrees of inflammation and neovascularization.

An alternative tracer recently evaluated for the assessment and characterization of atherosclerotic plaques is ${ }^{18} \mathrm{~F}$-labeled sodium fluoride $\left({ }^{18} \mathrm{~F}-\mathrm{NaF}\right)$. Preliminary studies suggest that ${ }^{18} \mathrm{~F}-\mathrm{NaF}$ uptake provides different information relating to metabolically active calcific plaques that are developing microcalcification (49). Prospective studies to determine the relationship between ${ }^{18} \mathrm{~F}-\mathrm{NaF}$ uptake, morphologic plaque characteristics, and future cardiovascular events are still required in subjects with stable and unstable coronary disease.

Although these new tracers still have to make their way into clinical use, several studies have reported the use of ${ }^{18} \mathrm{~F}$-FDG PET imaging as a means of characterizing inflammatory activity in atherosclerotic plaques, and thus as a surrogate biomarker for detecting vulnerable plaques (50).

Hybrid PET/MR imaging could also become the imaging modality of choice for the evaluation and follow-up of new therapeutic techniques aimed at tissue regeneration and myocardial repair. Recent studies have shown that PET imaging can be used for assessment of the kinetics of stem cell therapy in myocardial infarction. Dual labeling of stem cells with iron particles may offer new tools for monitoring the delivery, survival, and migration of cells after cell transplantation. However, the specificity of MR imaging and PET signals still needs to be validated in order to be applicable in cell therapy; therefore, hybrid PET/MR imaging is expected to play a major role in the validation process, allowing longitudinal studies that provide combined structural and biologic information.

\section{DISCUSSION AND CONCLUSIONS}

The integration of PET and MR imaging modalities in a single device will certainly open new perspectives in cardiac imaging by bringing together the 2 most advanced imaging technologies that provide competing but complementary information. Although MR imaging continues to expand in cardiology, becoming increasingly adopted in a clinical routine, the additional metabolic and functional information provided by PET tracers can provide valuable complementary data for specific subsets of patients who will benefit from the added diagnostic accuracy of PET. The first generation of coplanar PET/MR imaging devices that combined separate PET and MR imaging scanners led the way to new imaging protocols for the evaluation of myocardial viability and inflammatory cardiomyopathies. The more recent generation of integrated devices that allow the simultaneous acquisition of PET and MR images can provide the additional perspective of acquiring both modalities simultaneously after physiologic interventions such as pharmacologic stress. Integrated devices should allow the performance of rest and stress imaging protocols for the exploration of stress-induced ischemia in $\mathrm{CAD}$, showing both functional and biologic alterations in myocardial function. Early reports have shown the feasibility of combined PET/MR imaging protocols for the detection of CAD (5). The upcoming new generation of ${ }^{18} \mathrm{~F}$-labeled flow tracers will bring additional support for new PET applications in clinical practice in centers that do not benefit from a local cyclotron.

Further development of new tracers will expand the range of clinical capabilities of PET/MR imaging in cardiology, allowing more specific evaluation of vascular atherosclerosis and cardiomyopathy and the monitoring of new therapeutic tools for myocardial remodeling after acute ischemic events.

\section{DISCLOSURE}

No potential conflict of interest relevant to this article was reported.

\section{REFERENCES}

1. Flotats A, Knuuti J, Gutberlet M, et al. Hybrid cardiac imaging: SPECT/CT and PET/CT - a joint position statement by the European Association of Nuclear Medicine (EANM), the European Society of Cardiac Radiology (ESCR) and the European Council of Nuclear Cardiology (ECNC). Eur J Nucl Med Mol Imaging. 2011;38:201-212.

2. Schwitter J, Wacker CM, van Rossum AC, et al. MR-IMPACT: comparison of perfusion-cardiac magnetic resonance with single-photon emission computed tomography for the detection of coronary artery disease in a multicentre, multivendor, randomized trial. Eur Heart J. 2008;29:480-489.

3. Balyasnikova S, Lofgren J, de Nijs R, Zamogilnaya Y, Hojgaard L, Fischer BM. PET/MR in oncology: an introduction with focus on MR and future perspectives for hybrid imaging. Am J Nucl Med Mol Imaging. 2012;2:458-474.

4. Torigian DA, Zaidi H, Kwee TC, et al. PET/MR imaging: technical aspects and potential clinical applications. Radiology. 2013;267:26-44.

5. Rischpler C, Nekolla SG, Dregely I, Schwaiger M. Hybrid PET/MR imaging of the heart: potential, initial experiences, and future prospects. J Nucl Med. 2013;54:402-415

6. Ratib O, Nkoulou R, Schwaiger M. PET/MRI: a new era in multimodality molecular imaging. Clin Transl Imaging. 2013;1:10.

7. Levitt K, Guo H, Wijeysundera HC, et al. Predictors of normal coronary arteries at coronary angiography. Am Heart J. 2013;166:694-700.

8. Boden WE, O'Rourke RA, Teo KK, et al. Optimal medical therapy with or without PCI for stable coronary disease. N Engl J Med. 2007;356:1503-1516.

9. Pijls NH, Fearon WF, Tonino PA, et al. Fractional flow reserve versus angiography for guiding percutaneous coronary intervention in patients with multivessel coronary artery disease: 2-year follow-up of the FAME (Fractional Flow Reserve Versus Angiography for Multivessel Evaluation) study. J Am Coll Cardiol. 2010;56:177-184.

10. De Bruyne B, Pijls NH, Kalesan B, et al. Fractional flow reserve-guided PCI versus medical therapy in stable coronary disease. N Engl J Med. 2012;367:9911001 .

11. Nandalur KR, Dwamena BA, Choudhri AF, Nandalur MR, Carlos RC. Diagnostic performance of stress cardiac magnetic resonance imaging in the detection of coronary artery disease: a meta-analysis. J Am Coll Cardiol. 2007;50:13431353.

12. de Jong MC, Genders TS, van Geuns RJ, Moelker A, Hunink MG. Diagnostic performance of stress myocardial perfusion imaging for coronary artery disease: a systematic review and meta-analysis. Eur Radiol. 2012;22:1881-1895.

13. Fiechter M, Stehli J, Fuchs TA, Dougoud S, Gaemperli O, Kaufmann PA. Impact of cardiac magnetic resonance imaging on human lymphocyte DNA integrity. Eur Heart J. 2013;34:2340-2345.

14. Nael K, Fenchel M, Saleh R, Finn JP. Cardiac MR imaging: new advances and role of 3T. Magn Reson Imaging Clin N Am. 2007;15:291-300.

15. Schwitter J, Wacker CM, Wilke N, et al. MR-IMPACT II: Magnetic Resonance Imaging for Myocardial Perfusion Assessment in Coronary Artery Disease Trial: perfusion-cardiac magnetic resonance vs. single-photon emission computed tomography for the detection of coronary artery disease-a comparative multicentre, multivendor trial. Eur Heart J. 2013;34:775-781.

16. Ibrahim T, Nekolla SG, Schreiber K, et al. Assessment of coronary flow reserve: comparison between contrast-enhanced magnetic resonance imaging and positron emission tomography. J Am Coll Cardiol. 2002;39:864-870.

17. Sdringola S, Johnson NP, Kirkeeide RL, Cid E, Gould KL. Impact of unexpected factors on quantitative myocardial perfusion and coronary flow reserve in young, asymptomatic volunteers. JACC Cardiovasc Imaging. 2011;4:402-412.

18. Fiechter M, Ghadri JR, Gebhard C, et al. Diagnostic value of ${ }^{13} \mathrm{~N}$-ammonia myocardial perfusion PET: added value of myocardial flow reserve. $\mathrm{J} \mathrm{Nucl}$ Med. 2012;53:1230-1234.

19. Schindler TH, Nitzsche EU, Schelbert HR, et al. Positron emission tomographymeasured abnormal responses of myocardial blood flow to sympathetic stimulation are associated with the risk of developing cardiovascular events. J Am Coll Cardiol. 2005;45:1505-1512.

20. Sherif HM, Nekolla SG, Saraste A, et al. Simplified quantification of myocardial flow reserve with flurpiridaz F 18: validation with microspheres in a pig model. J Nucl Med. 2011;52:617-624.

21. Vermeltfoort IA, Raijmakers PG, Lubberink M, et al. Feasibility of subendocardial and subepicardial myocardial perfusion measurements in healthy normals 
with ${ }^{15} \mathrm{O}$-labeled water and positron emission tomography. J Nucl Cardiol. 2011;18:650-656.

22. Zaidi H, Nkoulou R, Bond S, et al. Computed tomography calcium score scan for attenuation correction of $\mathrm{N}-13$ ammonia cardiac positron emission tomography: effect of respiratory phase and registration method. Int J Cardiovasc Imaging. 2013;29:1351-1360.

23. Rees G, Bristow JD, Kremkau EL, et al. Influence of aortocoronary bypass surgery on left ventricular performance. N Engl J Med. 1971;284:1116-1120.

24. Schinkel AF, Bax JJ, Poldermans D, Elhendy A, Ferrari R, Rahimtoola SH. Hibernating myocardium: diagnosis and patient outcomes. Curr Probl Cardiol. 2007;32:375-410.

25. Beanlands RS, Nichol G, Huszti E, et al. F-18-fluorodeoxyglucose positron emission tomography imaging-assisted management of patients with severe left ventricular dysfunction and suspected coronary disease: a randomized, controlled trial (PARR-2). J Am Coll Cardiol. 2007;50:2002-2012.

26. Cleland JG, Calvert M, Freemantle N, et al. The Heart Failure Revascularisation Trial (HEART). Eur J Heart Fail. 2011;13:227-233.

27. Schinkel AF, Poldermans D, Elhendy A, Bax JJ. Assessment of myocardial viability in patients with heart failure. J Nucl Med. 2007;48:1135-1146.

28. Schröder O, Hör G, Hertel A, Baum RP. Combined hyperinsulinaemic glucose clamp and oral acipimox for optimizing metabolic conditions during ${ }^{18} \mathrm{~F}$-fluorodeoxyglucose gated PET cardiac imaging: comparative results. Nucl Med Commun. 1998;19:867874.

29. Abraham A, Nichol G, Williams KA, et al. ${ }^{18}$ F-FDG PET imaging of myocardial viability in an experienced center with access to ${ }^{18} \mathrm{~F}-\mathrm{FDG}$ and integration with clinical management teams: the Ottawa-FIVE substudy of the PARR 2 trial. J Nucl Med. 2010;51:567-574.

30. Patterson RE, Sigman SR, O'Donnell RE, Eisner RL. Viability assessment with MRI is superior to FDG-PET for viability: Con. J Nucl Cardiol. 2010;17:298-309.

31. Kato S, Kitagawa K, Ishida N, et al. Assessment of coronary artery disease using magnetic resonance coronary angiography: a national multicenter trial. J Am Coll Cardiol. 2010;56:983-991.

32. Eisentopf J, Achenbach S, Ulzheimer S, et al. Low-dose dual-source CT angiography with iterative reconstruction for coronary artery stent evaluation. JACC Cardiovasc Imaging. 2013;6:458-465.

33. Sharma OP, Maheshwari A, Thaker K. Myocardial sarcoidosis. Chest. 1993;103: 253-258.

34. Vignaux O, Dhote R, Duboc D, et al. Clinical significance of myocardial magnetic resonance abnormalities in patients with sarcoidosis: a 1-year follow-up study. Chest. 2002;122:1895-1901.

35. Takeda N, Yokoyama I, Hiroi Y, et al. Positron emission tomography predicted recovery of complete A-V nodal dysfunction in a patient with cardiac sarcoidosis. Circulation. 2002;105:1144-1145.
36. Yamagishi $\mathrm{H}$, Shirai N, Takagi M, et al. Identification of cardiac sarcoidosis with ${ }^{13} \mathrm{~N}-\mathrm{NH}_{3} /{ }^{18} \mathrm{~F}-\mathrm{FDG}$ PET. J Nucl Med. 2003;44:1030-1036.

37. Blankstein R, Osborne M, Naya M, et al. Cardiac positron emission tomography enhances prognostic assessments of patients with suspected cardiac sarcoidosis. J Am Coll Cardiol. 2014;63:329-336.

38. Nery PB, McArdle BA, Redpath CJ, et al. Prevalence of cardiac sarcoidosis in patients presenting with monomorphic ventricular tachycardia. Pacing Clin Electrophysiol. 2014;37:364-374.

39. Ohira H, Tsujino I, Ishimaru S, et al. Myocardial imaging with ${ }^{18} \mathrm{~F}$-fluoro-2deoxyglucose positron emission tomography and magnetic resonance imaging in sarcoidosis. Eur J Nucl Med Mol Imaging. 2008;35:933-941.

40. Maceira AM, Joshi J, Prasad SK, et al. Cardiovascular magnetic resonance in cardiac amyloidosis. Circulation. 2005;111:186-193.

41. Falk RH, Dorbala S. Pursuing an underdiagnosed disease: a simple imaging test for increasing suspicion of cardiac amyloidosis. Eur J Nucl Med Mol Imaging. 2011;38:467-469.

42. Antoni G, Lubberink M, Estrada S, et al. In vivo visualization of amyloid deposits in the heart with ${ }^{11} \mathrm{C}$-PIB and PET. J Nucl Med. 2013;54:213-220.

43. Chen W, Dilsizian V. Molecular imaging of amyloidosis: will the heart be the next target after the brain? Curr Cardiol Rep. 2012;14:226-233.

44. Ambrose JA, Tannenbaum MA, Alexopoulos D, et al. Angiographic progression of coronary artery disease and the development of myocardial infarction. J Am Coll Cardiol. 1988;12:56-62.

45. Stone GW, Maehara A, Lansky AJ, et al. A prospective natural-history study of coronary atherosclerosis. N Engl J Med. 2011;364:226-235.

46. Virmani R, Burke AP, Farb A, Kolodgie FD. Pathology of the vulnerable plaque. J Am Coll Cardiol. 2006;47:C13-C18.

47. Laitinen I, Saraste A, Weidl E, et al. Evaluation of alphavbeta3 integrin-targeted positron emission tomography tracer ${ }^{18} \mathrm{~F}$-galacto-RGD for imaging of vascular inflammation in atherosclerotic mice. Circ Cardiovasc Imaging. 2009;2:331338.

48. Gaertner FC, Kessler H, Wester HJ, Schwaiger M, Beer AJ. Radiolabelled RGD peptides for imaging and therapy. Eur J Nucl Med Mol Imaging. 2012;39(suppl 1): S126-S138.

49. Dweck MR, Chow MW, Joshi NV, et al. Coronary arterial ${ }^{18} \mathrm{~F}$-sodium fluoride uptake: a novel marker of plaque biology. J Am Coll Cardiol. 2012;59:15391548.

50. Cocker MS, McArdle B, Spence JD, et al. Imaging atherosclerosis with hybrid $\left[{ }^{18} \mathrm{~F}\right]$ fluorodeoxyglucose positron emission tomography/computed tomography imaging: what Leonardo da Vinci could not see. J Nucl Cardiol. 2012;19:12111225 . 